\title{
Systemic pigmented contact dermatitis to cobalt following ingestion of cobalamin supplement
}

\author{
Thanet Pongcharoensuk ${ }^{1}$ and Supitchaya Thaiwat ${ }^{1}$ \\ ${ }^{1}$ Phramongkutklao Hospital
}

February 15, 2021

\begin{abstract}
Systemic contact dermatitis (SCD) is a condition occurring in previously sensitized individuals after systemic re-exposure to the same or cross-reacting substance. Pigmented systemic contact dermatitis after intake of cobalt containing diet has never been reported.
\end{abstract}

\section{Introduction}

Cobalt is an essential element to humans in the form of cobalamin (vitamin B12). The human body contains approximately $1 \mathrm{mg}$ of cobalt, which around eighty percent is a component of vitamin B12. Human dietary intake of inorganic cobalt including green leafy vegetables, dairy products, liver and cereal grains varies between 5 and $50 \mu \mathrm{g} /$ day, while vitamin B12 represents cobalt only in minute amounts. Cobalt does not accumulate in the body and is rapidly excreted in urine. $(1,2)$

We report a case of systemic pigmented contact dermatitis, presenting as hyperpigmented lesions on face, neck and upper back caused by ingesting cobalt (cobalamin, vitamin B12). This patient had a history of ingesting cobalamin (vitamin B12) to treat her neuropathic pain 2 years earlier.

\section{Case description}

A 55-year-old non-atopic woman with an underlying condition of herniated nucleus pulposus presented slategrey hyperpigmented macules and patches on face, neck and upper back for 2 years (Fig. 1). She had a history of taking vitamin B12 supplement $600 \mu \mathrm{g}$ daily due to her neuropathic pain for 3 months before onset of hyperpigmented lesion. She denied having direct contact with cobalt, or any other metals and also agents which can induce slate grey hyperpigmentation including fragrances, rubber products or hair dye. Histopathology of the lesions showed focal vacuolar degeneration of the epidermis with superficial perivascular infiltration of lymphocytes and marked increase of dermal melanophages (Fig. 2).

Fig. 1 . Slate-grey hyperpigmented macules and patches on both cheeks

Fig. 2 . Histopathology from hyperpigmented lesions showed focal vacuolar degeneration of the epidermis and marked increase of dermal melanophages (black arrows) with superficial perivascular lymphocytic infiltration (H\&E x40, x100).

Systemic pigmented contact dermatitis was suspected, so patch testing was performed using the European Society of Contact Dermatitis guideline standard and cosmetic series (3). Readings were performed at 48 and 72 hours according to the International Contact Dermatitis Research Group criteria. Pigmented reactions to cobalt showed positive hyperpigmentation after 48 and 72 hours, while other standard and cosmetic allergens were negative (Fig. 3). We further performed tissue biopsy on the positive pigmented 
reaction. Histopathology showed focal parakeratosis with focal vacuolar interface dermatitis at the dermoepidermal junction and superficial perivascular infiltration with lymphocytes and melanophages consistent with pigmented systemic contact dermatitis similar to her previous active lesions (Fig. 4). The patient was advised to avoid taking oral vitamin B12. One year after diagnosis, the hyperpigmentation gradually faded after discontinuing cobalamin and adjunctive treatment with topical low potency corticosteroid and topical retinoic acid.

Fig. 3. Patch test read at 72 hours gave hyperpigmented reactions to cobalt (No.22).

Fig. 4. Histopathology from positive patch test pigmented reaction showed focal vacuolar degeneration of the epidermis with dermal melanophages (black arrows) with superficial perivascular lymphocytic infiltration $(\mathrm{H} \& \mathrm{E} \times 40, \mathrm{x} 100)$.

\section{Discussion}

Cobalt is an ingredient of vitamin B12 or cobalamin, reaction to cobalt may cause problems in patients receiving vitamin B12 replacement therapy. Several cutaneous manifestations have been reported in patients with allergic contact dermatitis to cobalt undergoing oral cobalamin replacement including atopic dermatitis, chronic vesicular hand dermatitis, cheilitis, and stomatitis. (4)

Systemic contact dermatitis (SCD) is a condition occurring in previously sensitized individuals after systemic re-exposure to the same or cross-reacting substance. Sensitization occurs due to varying exposures to antigens via multiple routes through the circulatory system, and thereby produces systemic allergic dermatitis, which may result in a variety of skin eruptions and systemic flare. The exact mechanisms behind SCD are not fully understood, but is thought to be a T-cell mediated delayed type hypersensitivity reaction. (5) The common allergens inducing systemic contact dermatitis include nickel, zinc and cobalt. (6-8) Several systemic agents are associated with pigmentation including cytotoxic agents, antimalarial, analgesics and silver but pigmented systemic contact dermatitis after intake of cobalt containing diet has never been reported.

Osmundsen et al. first described pigmented contact dermatitis (PCD) as a non-eczematous variant of contact dermatitis, clinically characterized by hyperpigmentation with little or no signs of dermatitis by observations that the melanosis was due to contact dermatitis caused by an optical whitener containing pyrazoline present in a washing powder. (9) The exact mechanism which allergens induce hyperpigmentation remains unknown. Some experimental studies have shown that allergens having a special affinity for melanin induced cutaneous inflammation increasing the number and size of the melanocytes and enhancing their enzymatic activity $(9,10)$. The common allergens responsible for pigmented contact dermatitis are listed in Table 1. Related studies have demonstrated cases of pigmented contact dermatitis and histopathological examination revealed focal vacuolar degeneration of the epidermis and increased dermal melanophages with superficial perivascular lymphocytic infiltration. $(11,12)$ Although the exact mechanism of how cobalt induces systemic contact dermatitis remains unknown (13), one related study revealed the increasing prevalence of cobalt allergy in the general population and the high popularity of food supplements containing cobalt, e.g., vitamin B12 supplement. (14)

\section{Conclusion}

We reported the case as first presenting hyperpigmented macules and patches on the face, neck and upper back after vitamin B12 ingestion. The results of the patch test with the cobalt also showed hyperpigmented reactions consistent with the clinical presentation suggesting the diagnosis of systemic pigmented contact dermatitis resulting from oral administration of cobalamin. The diagnosis was confirmed by histopathological results of the positive patch test results corresponding to the previous active lesions. We suggest investigating systemic pigmented contact dermatitis in reaction to cobalt when patients present unexplained hyperpigmented lesions.

Cosmetic Fragrances, Preservative, Hair dyes, Lipsticks

Fragrances Benzyl salicylate, ylang-ylang oil, Jasmine, Hydroxy-citronellal, Benzyl alcohol, Cinnamic alcohol, Lavender oi 


\begin{tabular}{ll}
\hline Cosmetic & Fragrances, Preservative, Hair dyes, Lipsticks \\
\hline Textiles & Azo dyes, Optical whiteners \\
Toiletries & Chromium hydroxide, Fragrances, Nickle oxide \\
Others & Minoxidil, Nickel sulfate \\
\hline
\end{tabular}

Table 1. Common allergens causing pigmented contact dermatitis

\section{Acknowledgement}

Published with written consent of the patients.

\section{Conflict of interest}

The authors have no conflict of interest to report.

\section{Author contributions}

TP: wrote the article, provided pathologic photography and features. ST: corrected the manuscript.

\section{Ethical approval}

We confirm that the manuscript has been read and approved by all named authors. The protection of intellectual property associated with this manuscript had been in our consideration.

\section{References}

1. Lison D. CHAPTER 25 - Cobalt. In: Nordberg GF, Fowler BA, Nordberg M, Friberg LT, editors. Handbook on the Toxicology of Metals (Third Edition). Burlington: Academic Press; 2007. p. 511-28.

2. MacPherson A, Dixon J. COBALT. In: Caballero B, editor. Encyclopedia of Food Sciences and Nutrition (Second Edition). Oxford: Academic Press; 2003. p. 1431-6.

3. Johansen JD, Aalto-Korte K, Agner T, Andersen KE, Bircher A, Bruze M, et al. European Society of Contact Dermatitis guideline for diagnostic patch testing - recommendations on best practice. Contact Dermatitis. 2015;73(4):195-221.

4. Brescoll J, Daveluy S. A review of vitamin B12 in dermatology. Am J Clin Dermatol. 2015;16(1):27-33.

5. Jacob SE, Zapolanski T. Systemic contact dermatitis. Dermatitis. 2008;19(1):9-15.

6. Zirwas MJ, Molenda MA. Dietary nickel as a cause of systemic contact dermatitis. J Clin Aesthet Dermatol. 2009;2(6):39-43.

7. Panaszek B, Nowak D, Cieślik K, Dziemieszonek P, Gomułka K. Systemic contact dermatitis caused by cobalt chloride and palladium in a 26-year-old woman with allergic type I reactions, non-steroidal antiinflammatory drug hypersensitivity and autoimmune thyroiditis. Postepy Dermatol Alergol. 2017;34(4):38890.

8. Yoshihisa Y, Shimizu T. Metal allergy and systemic contact dermatitis: an overview. Dermatol Res Pract. 2012;2012:749561.

9. Osmundsen PE. Pigmented contact dermatitis. Br J Dermatol. 1970;83(2):296-301.

10. Papa CM, Kligman AM. The behavior of melanocytes in inflammation. J Invest Dermatol. 1965;45(6):465-73.

11. Shin J-W, Choi J, Huh C-H, Na J-I. Two Cases of Pigmented Contact Dermatitis Caused by Pure Henna Hair Dyes. Annals of Dermatology. 2018;30:735. 
12. Serrano G, Pujol C, Cuadra J, Gallo S, Aliaga A. Riehl's melanosis: pigmented contact dermatitis caused by fragrances. J Am Acad Dermatol. 1989;21(5 Pt 2):1057-60.

13. Arslan S, Aksan S, Ucar R, Caliskaner AZ. Contact dermatitis to cobalt chloride with an unusual mechanism. Prosthet Orthot Int. 2015;39(5):419-21.

14. Thyssen JP, Menne T. Metal allergy-a review on exposures, penetration, genetics, prevalence, and clinical implications. Chem Res Toxicol. 2010;23(2):309-18.
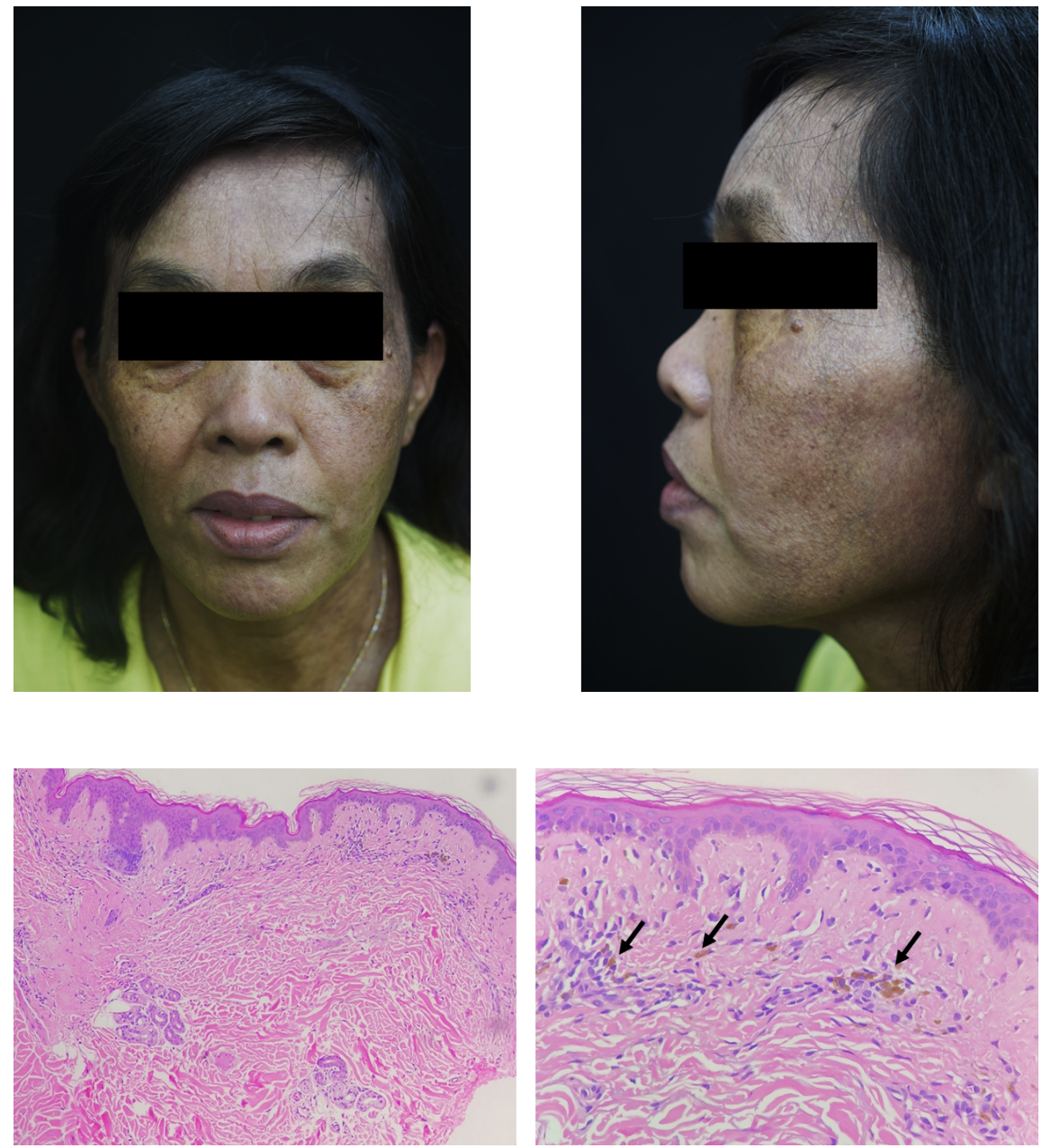

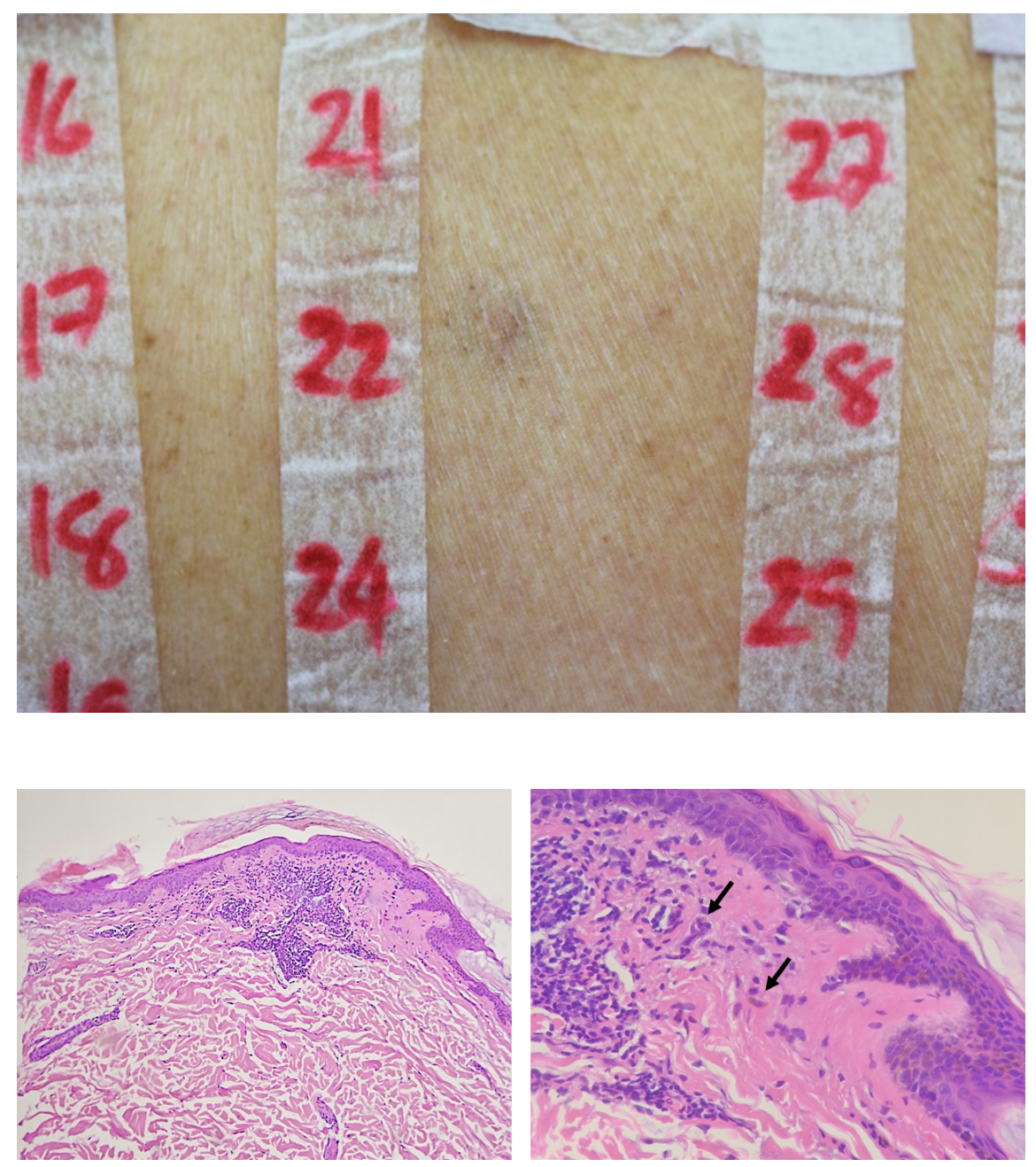